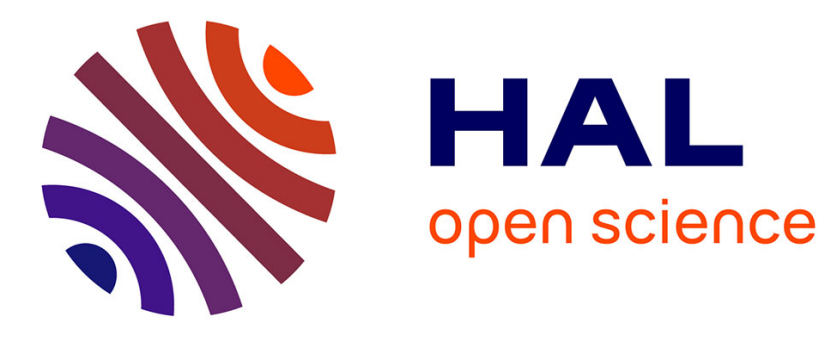

\title{
New Atlantis: Audio Experimentation in a Shared Online World
}

Peter Francis Sinclair, Roland Cahen, Jonathan Tanant, Peter Gena

\section{To cite this version:}

Peter Francis Sinclair, Roland Cahen, Jonathan Tanant, Peter Gena. New Atlantis: Audio Experimentation in a Shared Online World. Mitsuko Aramaki, Richard Kronland-Martinet, Sølvi Ystad. Bridging People and Sound. 12th International Symposium, CMMR 2016, São Paulo, Brazil, July 5-8, 2016, Revised Selected Papers, 10525, Springer, pp.229-246, 2017, Lecture Notes in Computer Science, 978-3-319-67738-5. 10.1007/978-3-319-67738-5_14. hal-01791975

\section{HAL Id: hal-01791975 \\ https://hal.science/hal-01791975}

Submitted on 22 Jun 2018

HAL is a multi-disciplinary open access archive for the deposit and dissemination of scientific research documents, whether they are published or not. The documents may come from teaching and research institutions in France or abroad, or from public or private research centers.
L'archive ouverte pluridisciplinaire HAL, est destinée au dépôt et à la diffusion de documents scientifiques de niveau recherche, publiés ou non, émanant des établissements d'enseignement et de recherche français ou étrangers, des laboratoires publics ou privés. 


\title{
New Atlantis: Audio Experimentation in A shared Online World
}

\author{
Pete Sinclair ${ }^{1}$, Roland Cahen ${ }^{2}$, Jonathan Tanant ${ }^{3}$ and Peter Gena ${ }^{4}$ \\ ${ }^{1}$ Ecole Superieur d'Art d'Aix. Locus Sonus Research Unity. Aix-En-Provence, France \\ ${ }^{2}$ Ecole Nationale Superiere de Creation Industrielle. (ENSCI les ateliers), Paris France. \\ ${ }^{3}$ Independant Software Engineer, Jon Lab. Tilly, France. \\ ${ }^{4}$ School of the Arts Institute Chicago. (SAIC) Chicago, USA. \\ peter.sinclair@ecole-art-aix.fr \\ roland.cahen@ensci.com \\ jonathan@free.fr \\ pgena@artic.edu
}

\begin{abstract}
Computer games and virtual worlds are "traditionally" visually orientated, and their audio dimension often secondary. In this paper we will describe New Atlantis a virtual world that aims to put sound first. We will describe the motivation, the history and the development of this FrancoAmerican project and the serendipitous use made of the distance between partner structures. We explain the overall architecture of the world and discuss the reasons for certain key structural choices. New Atlantis' first aim is to provide a platform for audio-graphic design and practice, for students as well as artists and researchers, engaged in higher education art or media curricula. We describe the integration of student's productions through workshops and exchanges and discuss and the first public presentations of NA that took place from January 2016. Finally we will unfold perspectives for future research and the further uses of New Atlantis.
\end{abstract}

Keywords: audiographic creation, audio for virtual environments, sound spatialisation, networked music.

\section{Introduction}

New Atlantis is a shared (multi-user) online virtual world dedicated to audio experimentation and practice. Unlike most online worlds where image is the primary concern, in NA sound comes first. NA provides a context for new-media students to showcase research projects that explore the relationship between sound, virtual 3D image and interactivity. It offers a pedagogical platform for audiographic animation, real-time sound synthesis, object sonification and acoustic simulation. It is a place to organize virtual sound installations, online concerts, Soundwalks and other audiovisual art experiences. 
The name New Atlantis comes from the title of an unfinished 1628 utopian novel by philosopher Francis Bacon [1], which describes a legendary island somewhere in the ocean, doted with extraordinary audio phenomena that might be considered as premonitory of today's electronic and digital audio techniques. We have adopted some of Bacon's ideas and nomenclature to create classes for the virtual world such as "Sound Houses", "Sound Pipes", "Trunks" and "Helps". In NA all elements are intended to have audio qualities: spaces resonate, surfaces reflect and collisions activate the multiple sounds of the objects involved. A collection of purpose built scripts implement low level sound synthesis and multiple parameter interactivity, enabling the creation of complex sound sources and environments linked to animation or navigation in the visual scene.

NA can be accessed via a web viewer or as a standalone application. It is organized as "spaces" that can be accessed independently but that share the same basic principles of navigation and specific scripts. Multi-user, it can be shared by several players at the same time making it suitable for group playing in both the gaming and the musical sense of the word. Every registered user can create and host individual or shared spaces which he or she can decide to make persistent or not. At the time of writing, we are working on a limited number of public "spaces" that contain multiple "Sound Houses" (architectural elements with specific acoustics) and other sound objects. These can be visited by navigating through the scene or created within the scene. The audio "mix" of these different sources varies with distance, so placing and navigating between sound objects can become a musical experience. In public spaces, players can interact with one another and with shared objects potentially playing together.

New Atlantis project is not only about creating a multi user virtual universe, but also about making it together while learning. It is experimental, creative and educational. New opportunities for further development in NA are created through the organization of workshops, courses or events that group art students in different locations, working together at a distance. It is a way to encourage ubiquitous working groups of students to share immaterial and non-local (international) art projects. Most sound and music education schemes tend to be oriented towards established disciplines. NA on the other hand encourages experimental design projects and the exploration of new or emerging creative fields. Francis Bacon's New Atlantis proposed a model for the role of science and art in society that placed education at the heart of culture. Our project emphasizes discovery, cultural exchange, experimentation, learning and furthering of knowledge in an educational and creative environment. 


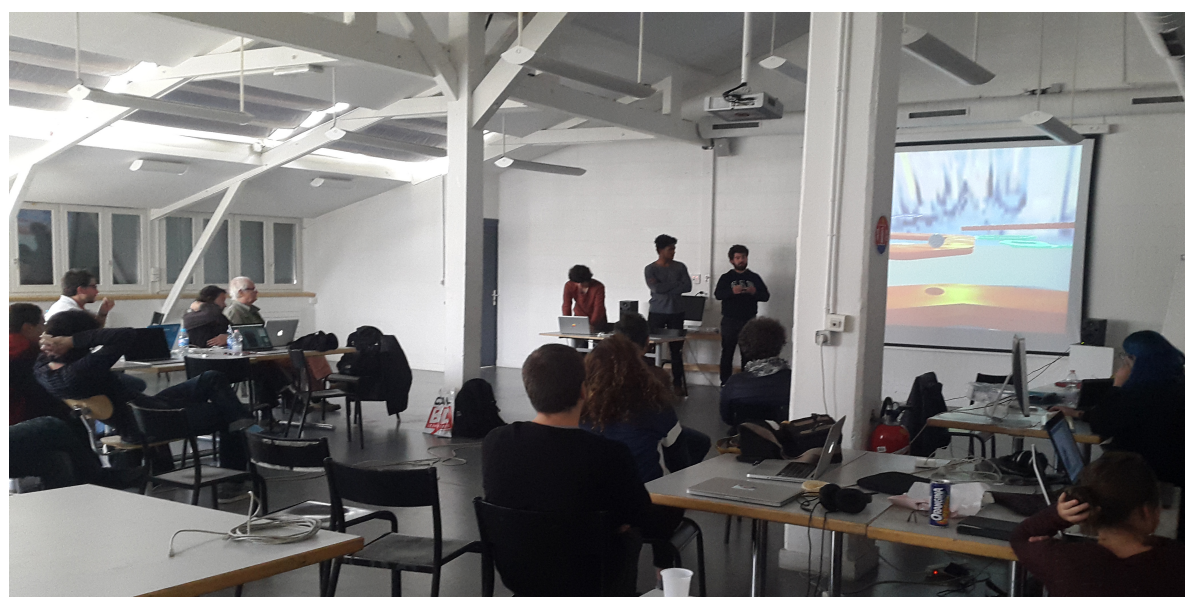

Fig 1. Students showcasing their projects in New Atlantis. ENSCI les ateliers, September 2015.

\section{Context and History}

"We have also sound-houses, where we practise and demonstrate all sounds and their generation. We have harmony which you have not, of quartersounds and lesser slides of sounds. Divers instruments of music likewise to you unknown, some sweeter than any you have; with bells and rings that are dainty and sweet. We represent small sounds as great and deep, likewise great sounds extenuate and sharp; we make divers tremblings and warblings of sounds, which in their original are entire. We represent and imitate all articulate sounds and letters, and the voices and notes of beasts and birds. We have certain helps which, set to the ear, do further the hearing greatly; we have also divers strange and artificial echoes, reflecting the voice many times, and, as it were, tossing it; and some that give back the voice louder than it came, some shriller and some deeper; yea, some rendering the voice, differing in the letters or articulate sound from that they receive. We have all means to convey sounds in trunks and pipes, in strange lines and distances." [1]

The origins of the NA project go back to 2005 when the Locus Sonus, ESA-Aix (Ecole Superieur d'Art d'Aix-En-Provence) and SAIC (School of the Art Institute of Chicago) were awarded FACE[2] funding for an academic and research exchange program. The original impulse occurred through collaboration between teams of the $3 \mathrm{~d}$ and sound departments of the partner establishments, which led to the observation that there was scope for research into the area of audio in games and other virtual environments.

That NA refers to a Utopian model can be interpreted in several different ways. Firstly as the above citation demonstrates, the original text by Francis Bacon 
describes an island territory that was home to numerous extraordinary audio phenomena. Beyond this the novel predicts the principles of the contemporary research university as seen in the description of "Salomon's House" which promotes international exchange: "For the several employments and offices of our fellows, we have twelve that sail into foreign countries ... who bring us the books and abstracts, and patterns of experiments of all other parts" [1] and trans-disciplinary research: "We have three that collect the experiments of all mechanical arts, and also of liberal sciences, and also of practices which are not brought into arts"[1]. These ideas, combined with the fact that we are indeed engaged in creating a utopian world that might be considered as situated (albeit in our imagination) somewhere in the ocean between Europe and America - combine to make NA a suitable reference.

Since the ESA-Aix/SAIC partnership was separated by the Atlantic Ocean, we rapidly adopted networked solutions for our collaborations: video conferencing and remote desktop were used to exchange lessons and conferences and an interconnected $3 \mathrm{~d}$ cave were set up with an interface in both Aix and Chicago. The first experiments in 3d audio-graphy took place in Second Life[3], using Pure Data[4] as an audio engine. The process involved sending html commands from second life to an external server that did the audio synthesis and streamed the result back to second life; the project was presented in 2009 at the "Second Nature" festival in Aix-En-Provence[5]. This system worked well enough to convince us that it was worthwhile pursuing the development of sophisticated audio features for virtual environments, however, it was difficult to implement and the delay due to streaming was problematic.

The decision was made to build our own multi user world using Panda 3d[6] with Pure Data[4] bundled as an audio engine. Ecole Nationale Superieure de Creation Industrielle (ENSCI, les ateliers) became associated with the project at this point. This first version of NA was developed during multiple workshops that took place in Chicago and Aix en Provence between 2007 and 2011. The project involved a relatively complex path finding system used to calculate acoustics and custom-built client server software [7]. Although a working version was tested successfully during a workshop at ENSAB in 2011[8], it was decided to abandon the development based on Panda/Pd in favor of Unity3d[9], a more recent and efficient platform offering greater scope for audio programming and possessing built in networking capabilities.

\section{New Atlantis Project Aims}

There are multiple aims associated with the NA project: as mentioned above the project emerges from an international exchange program and one of its first ambitions is to provide a platform for international academic, artistic, cultural and scientific exchange. The more specific aim is to further research into audio for virtual environments with the precise goals of providing an educational tool for students and a synchronized platform for remote music and sound art practices. 


\subsection{Research Into Audio for Virtual and networked Environments, background.}

The history of audio development in game environments is relatively short and, at least when this project was initiated, somewhat lacking in substance. Arguably, this might be put down to competition between audio and visual requirements in terms of processing power on personal computers or game boxes. If in recent years companies such as AudioGaming[10] that are specialized in audio for game environments have started to appear, for the essential they provide sound design services for the commercial game market, rather than considering the virtual world as a possible audio interface. Therefore the historical origins of this project might be considered from several other angles. One approach is that of visual interfaces for musical compositions such as UPIC[11] originally developed by Iannis Xenakis or navigable scores such as Fontana Mix 1958 by John Cage[12]. Distant listening, remote performance and other streamed and networked art forms are another thread that has been largely investigated by Locus Sonus[13]. Experiments with remote musical presence started as early as 1992 when during an event organized by Michel Redolfi; Jean Claude Risset and Terry Riley in Nice played with David Rosenboom and Morton Subotnick in Los Angeles using Disklaviers and a satellite connection[14]. In 1967, the late Maryanne Amacher conceived of and produced City Links, in Buffalo, NY. The 28-hour performance took live microphone feeds from five different locations in the city. Victor Grauer and Max Neuhaus were involved in the Buffalo production and Neuhaus subsequently did similar work that came to be known as "telematic performance". The rapidly developing discipline of sonification[15] is equally useful when reflecting on the sound of virtual objects. In effect the different members of the research team have pursued these different lines of investigation over the past decades.

We should not however ignore early forays into audio-graphic creation an example being Ivan Chabanaud ${ }^{1}$ and co-author Roland Cahen's Icarus [16]. When this virtual reality project was started in 1995, sound synchronization techniques where cumbersome: a Silicon graphics machine rendering visual objects was connected to Opcode Max 3 (MIDI only) using an external MIDI expander and the IRCAM's spatialisation system. More recent audio-graphic projects that $\mathrm{R}$. Cahen has been involved in include BANDONEON[17], PHASE[18], ENIGMES[19], and TOPOPHONIE[20].

Other recent research initiatives focusing on shared virtual environments such as UDKOSC[21] use the OSC protocol to associate external audio engines such as Pd or Supercollider with a virtual environment. Although the original version of NA

\footnotetext{
${ }^{1}$ Ivan Chabanaud, was a fascinating french digital artist who gave wings to his audience as they became icarus for the space of a visit. Our friend Ivan died the $4^{\text {th }}$ september 2015.
} 
followed this line of investigation we have switched to the using the audio engine incorporated in Unity for the sake of simplicity and versatility on the user side (see section 6.). The Avatar Orchestra Metaverse, formed in 2007, is another Interesting experimental project led by American Sound Artist, Pauline Oliveros: "The Avatar Orchestra Metaverse is a global collaboration of composers, artists and musicians that approaches the virtual reality platform Second Life as an instrument itself'[22]. However this approach is very different to that adopted for New Atlantis in the sense that it is dependent on the resources of the existing virtual world Second Life. Proteus by Ed Key and David Kanaga [23] is a rare example of a virtual world where the combination of low-resolution graphics and generative composition create an everevolving landscape/soundscape, offering a delicate interaction between the audio and visual components.

\subsection{Fields of Investigation}

\section{Interactive sound and music with virtual objects}

Audiographic design is a multimodal approach that consists of coordinating graphical form and behavior with auditory events in the design of virtual objects. These objects, although they are visual images, can incorporate physical simulation so they can interact with us and between themselves. While artists and designers often have a limited culture of multimodality, creating audiographic objects or compositions requires interconnections between various domains of expertise such as sound design, graphic design, 3D animation, real time interaction and coding. New Atlantis has been conceived to provide an adequate platform for this type of training. Designing multimodal interactions and implementing them in a virtual world is more complex than creating simple visual representations or video. It obliges the author to resolve more design issues and to give objects deeper consistency, bringing them close to a physical reality, even if they remain immaterial. At a certain point, the simulated object becomes the object in its own right and the notion of "virtual" is modified. Arguably, virtual objects are somehow non-material objects that incarnate their own reality. This has long been the case in music as well as with other abstract artistic activities, when an initial manipulation of representation ceases to be the finality.

Since the audiographic relationship is constructed, it can also be fictitious. An object's sound can be modified to become quite different from the "real life" original, while paradoxically appearing very real or possibly more than real (hyperreal). An early example of this phenomenon can be found in Jacques Tati's film Mon Oncle where "retouched" audio recordings focus attention unnaturally on specific banal visual objects [24]. We consider that this bending, schematizing or detournement of the relation between the visual object and its associated sound is a fruitful terrain for investigation. Virtual composition also allows us to interact with sound objects using a variety of expressive devices such as physical modeling, avatar form and behavior, fictional scenarios and gameplay, opening a multitude of new forms of narration. 
We are currently developing new tools for New Atlantis in collaboration with FRE PRISM that will incorporate LMA's intuitive synthesizer control system. The system is based on a principal of control by metaphorical description of material qualities and physical actions. It is thus highly suitable for the creation of virtual objects in particular since the simulated object does not necessarily require a real-world model.

\section{Spatialization, Sound Navigation and Virtual Acoustics}

Sound navigation consists of browsing through different sound sources placed in a spatialized sound scene, thereby composing a musical form from the mix produced by movements relative to these sources. It is an artistic transposition of our sound experience in the physical world [25]. In virtual worlds, sounds are mainly spatialized through orientation and distance. The simple act of navigation allows us to create spatial mixes or canons as well as subject and object motion sequences. Spatial acoustics are another important part of our audio perception and indeed they participate in our natural audio interactions as we activate reverberant spaces through our own actions such as footsteps or vocalizations [26]. This praxeology[27] of sound space can also be developed in virtual environments and the juxtaposition of different acoustic responses as we navigate while generating sounds or listening to sounds generated by other players, provides an original approach to real-time audio signal processing. Short simple sound sources such as clicks or collision sounds can be used to activate and compare virtual acoustics and continuous sound sources can be multiplied to create complex harmonics. Such indeterminate forms of navigation, inspired by SoundWalking or other Soundscape related activities (see The Tuning of the World by R.Murray Schafer [28]), are alternatives too more permanently authored audiographic forms.

\subsection{A Synchronized Platform for Remote Music and Sound Art Practices}

\section{Networked Playing and Performance.}

In the case of NA "playing" may mean "gameplay" i.e. in general video games parlance "the specific way in which players interact with a game"[29], or it can equally be used to designate musical playing. In the preceding section, we approached NA as a compositional tool but it can also be considered as an audio "sandpit", as a shared instrument, or as a stage for public performances. The fact that these activities are synchronized online means that NA also opens a line of investigation into remotely shared musical and sound art practices. This in turn invites speculation as to the possibility of a creating a new paradigm in musical practice and distribution, a question that we wish to investigate in future research.

Networked music performances have existed since the beginning of the Internet and even before through the use of telephone and radio (see examples in section 3.1.). Until now however, such activities have, for the most part, focused on mixing networked (streamed, midi or OSC controlled sounds) with locally sounding instruments an example is Jean Claude Risset's use of the Disc Clavier, another is 
French musician Eric M's practice of using Locus Sonus' “open microphone" live audio streams [30] to introduce indeterminate sounds into his DJ sets. With NA however, the virtual sound space is distributed, and co-users share events, functions, triggers, controllers and audio recordings (made on the fly) through a dedicated server. The fact that all connected users share the "current state" of a space (see technical explications in section 3) means that as long as an instance of a space is active, they experience the same evolving situation from wherever they are (within the limits of the network's capabilities). There is no distinction per se between musician and audience, this allows for inventive approaches to shared performance (for example, if one player introduces a sound source object or makes a recording, other players can hear it, move it or modify its parameters).

\subsection{An Educational Tool}

Locus Sonus[31] is a research unit based at ESA - Aix and funded by the French ministry for culture. Its main concern is practice-based research into sound art. Locus Sonus' research investigates virtual and acoustic sound space in a permanent exploration of "new auditoriums"[13]. ENSCI les Ateliers, have worked on numerous sound design projects including sounding objects, industrial sound design, audiography, sound spatialization, auditory interfaces but also sound art, electronic music, radio broadcasts, soundtracks for films, and various research projects [32]. The School of the Art Institute of Chicago's Art and Technology department teach virtual and augmented reality, gaming, $3 \mathrm{~d}$ modeling, computer imaging, live performance audio and immersive audio[33]. Between them, these three structures provide the multidisciplinary expertise, creative and educational context that the NA project is founded on. The recent addition of the FRE PRISM as a new partner adds elements of fundamental research in the domain of digital audio to this rich consortium.

\section{Teaching Sound in Art and Design Schools}

Over the last 20 years sound education in France has progressively shifted from the conservatoires (music academies) to schools of art and design. The main reason for this is that, apart some rare exceptions, conservatoires were designed to teach solely classical music theory and instrumental practice and have therefore experienced difficulty in adapting to the challenges of XXth and XXIst century music and sound art production. As a consequence sound arts, sound design, some performing arts and sound for multimedia are now taught in many art and design schools including ENSCI and ESA-Aix. Most of the students concerned are consequently visual artists and rarely trained musicians. On the one hand this is a drawback, because they have a limited sonic culture, and on the other it is an advantage because they don't have preconceived models or cultural bias. Similarly, unlike traditional composers who arguably consider music as a result of relationships between pitch and rhythm, artists tend to use sound as material. Since their culture is primarily visual, these students 
have a tendency to create sounds in relation to images and to be concerned with multimedia and interaction.

\section{Working and Creating Online in Virtual Workshops, Complementary Skills \& Delocalized Working Groups}

Creating and playing music together and by extension audiographic composition and performance are a great way to link people; this holds for artists, designers and students as well as the game audience. NA "bridges people with and through sound" since it is a group project within which participants with different skills and different degrees of expertise can come together and cooperate. Thus although the permanent team of NA is constituted of experienced developers, researchers, artists and teachers, this team has been completed from the outset of the project by successive generations of post graduate students. The architecture of NA has been designed in such a way as to encourage a variety of artistic approaches and methodologies, a creative participant can easily build a new and unique space (or level) however different propositions can also be easily combined into a single space. This method of creating content is gradually enlarging and enriching the scope of the project through a process of experimentation, feedback and exchange. On another level, as described above, creating content for the world is also be a challenging pedagogical exercise. Students are encouraged to work in teams pooling skill sets and sharing experience. The NA team is spread over several geographic locations, rather than this becoming a handicap it has turned into a fundamental characteristic of the project. NA is progressively becoming the place where we meet to work and remote collaboration is in the process of becoming second nature.

\section{$4 \quad$ Architecture and Development}

The particular aims of New Atlantis, described above, imply that certain decisions have been made regarding the architecture and other parameters of the world. To give some examples: the world needs to be shared online, it should be easy to use by a wide range of players (including those creating content) and audio performance including synchronization between players - is paramount.

\subsection{System Architecture}

New Atlantis consists of three main software components:

The first is a MySQL database / PHP backend. The second is a standalone app (Mac OS X/Windows), named the Viewer, which allows the player to navigate the world, host multiplayer sessions, manage his account (spaces, assets...) and upload content. The third (optional) component is the SDK, which allows the advanced participant to build a viewer and to create components and contents using Unity3D authoring software. 


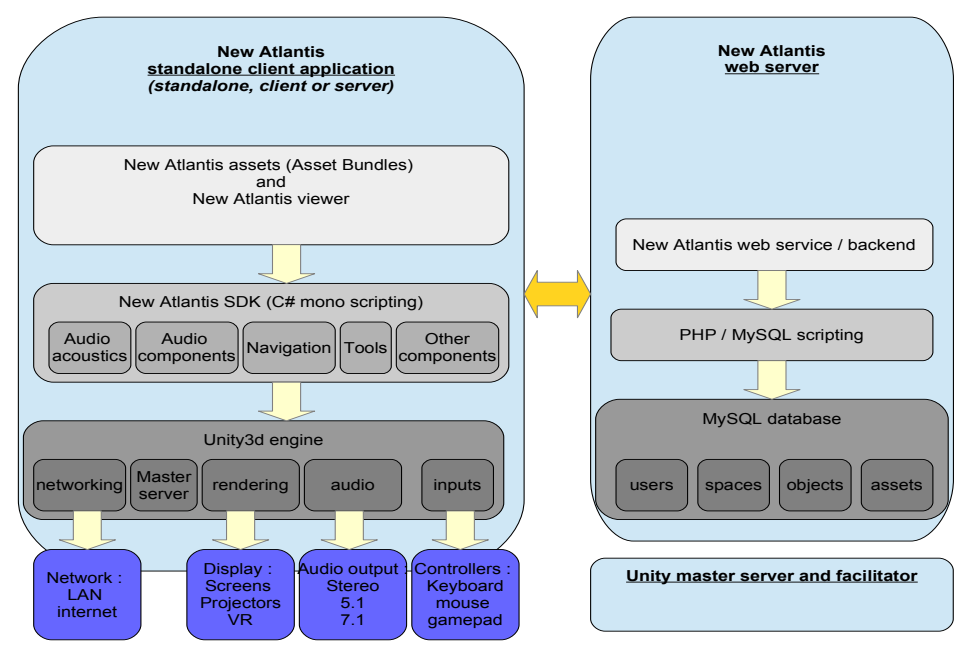

Fig 2. System architecture overview.

\section{Entities and Related Concepts}

A Space is an independent New Atlantis world. It is not necessarily related to the Bacon Sound Houses concept: several Sound Houses could exist in one Space, or one Sound House could be split in several Spaces. The absolute rule is that one Space is totally independent: nothing communicates with, exits to or enters from the outside.

An Object is a composite-audio-graphic-3d-interactive object created by a participant (designer/artist/developer) in Unity and uploaded to a Space in the form of an "Asset Bundle". Objects have qualities (in particular audio capabilities) and consist of a data packages with associated states. These include the updating position, orientation, and other related parameters in the currently activated space. When not in a space, an object is referred to as an asset.

A player (user or visitor) is a human user of the New Atlantis platform. The system holds parameters related to the players, such as email, login, password and a list of assets.

The viewer is the multi-purpose application that is the main tool a player has to use to visit and navigate a space.

\subsection{Synchronization and Data Persistence}

As a shared audio world, New Atlantis has stringent needs when it comes to synchronization since, as described above, we wish players to be able to play together musically. This requires that what each player hears on different connected computers is as close as is possible to the same thing. 


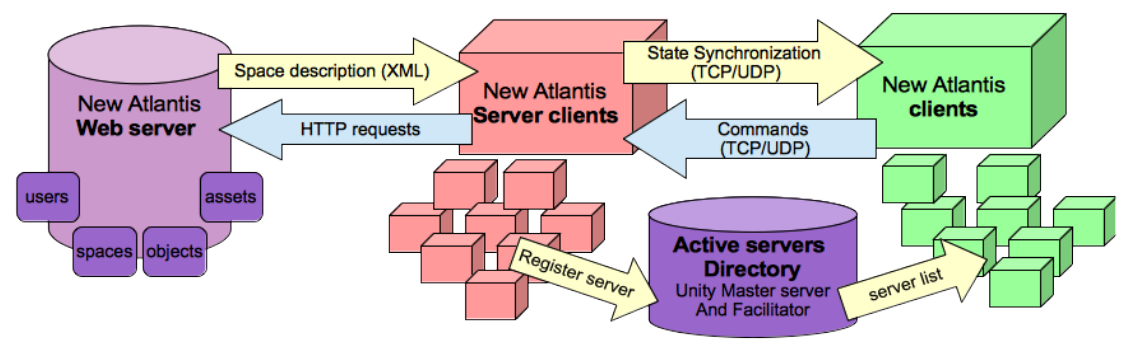

Fig 3. Synchronization and data persistence architecture overview.

A MySQL database / PHP backend holds the main state of objects and a web service allow the access to this database, including creation, modification and deleting of objects. Any player is able to host a session on his/her personal computer and accept other players running the same viewer application, from virtually anywhere in the world (within the limits of firewall and network structures). This is implemented using Unity's built-in advanced network features (NAT punchthrough, Master server, Facilitator server) - so instead of running a dedicated server, we decided to take this simpler and more flexible approach where any user is able to host a New Atlantis session with a chosen Space (this method is used by most multiplayer video games).

We have had to define and implement the way Unity's network system manages synchronization, when several players are connected together in a session. We decided to use an authoritative server scheme, meaning that the server runs the entire simulation and sends objects states to the clients that are running the scene without the logic (solely the rendering). The only exception to this is the avatar position, which is not sent by the server but generated by each client to guarantee good local fluidity (to avoid lag in the avatar's own position). The number of objects that need to be synchronized has a direct effect on performance and we tried several variations on "depths" of synchronization, from every single object to only a few main ones. There is inevitably a compromise that has to be made between performance and accuracy in simultaneity between clients (largely dependent on bandwidth). We should insist here on the special needs of working with sound: principally good temporal synchronization. To sum up, in the viewer application, two types of synchronization occur:

1. A synchronization of the objects contained in a given space: the viewer app connects to the web service and downloads an XML manifest (list) of all objects in the space with their initial state (position, orientation, name). Each object is an Asset Bundle that is then downloaded by the viewer and held in cache for future retrieval. 
2. A client/server synchronization with Unity Network engine: synchronization of avatars positions, created objects, audio recorded during playing... The Unity Network engine implementation works with TCP/IP and UDP/IP messages sending with a proprietary format including synchronized parameters serialization. We have had to implement RPCs (Remote Procedure Calls) functions to handle special cases and audio-graphic synchronization.

\subsection{Content Creation: Tradeoff Between Simplicity and Features}

As described in section 2, NA has several levels of usage. Technically, the system needs to be used by a wide range of players with skills that range between basic gaming and professional design and programming. Advanced players, such as students in workshops, can use the NA SDK package in Unity, while a player without such skills is still be able to run the viewer app and access the spaces without doing any programming. It should be mentioned that some of the students during the workshops and throughout the duration of project development have even programmed substantial improvements and add-ons to the platform.

We have identified the following users:

1. Simple player/visitor - visits and navigates a space.

2. Advanced player/visitor - creates spaces with existing assets.

3. Builder - builds new assets to be used and shared.

4. Core developer - creates new standard NA components.

Unity is used both as a content creation platform and as the game engine. This means that while it is tempting to allow the builder to use Unity's authoring tool with as few restrictions as possible, it is necessary to forbid practices that could lead to issues, such as bad synchronization or malfunctioning components.

\section{The need to standardize accepted scripts}

For security and design reasons, builder created scripts cannot be included in Asset Bundles. User-created Objects can reference existing scripts, but a new script (at the time of writing), needs to be included in the viewer to be available in the system. A workaround would be to build a .NET assembly using Mono Develop or Microsoft Visual Studio and use .NET reflection to make it available at runtime. However this is still in development and is not a perfect solution since it would also enable potentially unlimited access to scripting with the implied security problems.

\section{Performance considerations}

Resources are a major concern when it comes to user-generated content: a player could potentially build a highly complex object that is too "greedy" with the resources 
of visiting computers (including video rendering performance, download time, CPU processing and memory). We have decided to manage this in two ways. Firstly, by defining "good practice" guidelines, these include: limiting the number of simultaneously playing sound sources; reducing the number of physical collisions and rigid bodies; reducing the size of audio clips; avoiding triggering too many audio sources too frequently; being careful with the use of custom DSP processing. In addition to these guidelines, we have limited the Asset Bundle upload size to a maximum of $7 \mathrm{MB}$. The second approach is via the implementation of active optimization schemes, such as dynamic audio sources management as well as dynamic components (distant audio sources and objects in general are deactivated).

\subsection{The Viewer App and the Standard Tools and Navigation}

As we aim for high coherency between spaces and the player's experience, we decided to introduce some standard concepts.

1. Navigation: after a few early discussions and tests concerning a userdefinable avatar and navigation system, we finally decided to provide a standard navigation system, that includes a few cameras and standard controls (with keyboard and mouse or with a dedicated gamepad).

2. Interactions and tools: we designed all interactions as "tools", that are selectable in the viewer by the player. A large number of these tools have been suggested many of which concern variations on modes of audio interaction (rubbing or dragging as well as simply colliding). At the time of writing roughly half of these ideas are implemented including sound-playing tools, an object thrower, physical interactions, flashlight, sunlight, trunk creation (audio recorder)...

\section{Audio Components}

We have built a library of audio components to be used on audiographic objects in New Atlantis, these include:

1. Audio synthesis: noise generation, oscillators, FM synthesis, loopers, wave terrain synthesis...

2. Audio triggering: play/stop an audio source based on specific events, such as a collision, a volume intersection (named a trigger), a threshold distance with the listener...

3. Audio parameter modulation: pitch, volume, panning...

4. Experimental audio filtering: implementation of Bacon's audio helps.

5. Audio recording: with content synchronization over the network (the "trunk").

This list of audio components continues to augment with the ultimate intention of providing a comprehensive toolbox for DSP interaction and sound generation. 


\section{Other standard components}

Because in New Atlantis designers can only use built-in scripts, it has also been necessary to provide a wide range of other standard components, including animations, visuals, move and rotate, teleport, GUI, particles triggering, physics...

\subsection{Audio Spatialisation and Virtual Acoustics}

A standard approach to audio spatialisation divides perception into three classes: the direct path, the early reflections and the late reverberation. As most 3D game engines today, Unity has a simple 3D audio sources management, which we used to spatialize an audio source's direct path. Unity also has a concept of Reverb zones with dedicated parameters (such as presets, level, reverb time....), but the Reverb effect is applied to the final mix and is triggered by the listener position - this means that by default, it is not possible to have sources in different spaces and have each source /space resonate independently since all sources are reverberated simultaneously. To compensate for this we have introduced the possibility for an Audio source to make its immediately surrounding space resonate (NAReverbEffector / Resonator) by using volume triggering, this allows us to have more complex shapes than standard Unity reverb zones (simple spherical volumes). This is a workaround since instead of the listener it is the audio source that is reverberated (we found this to be a better compromise).

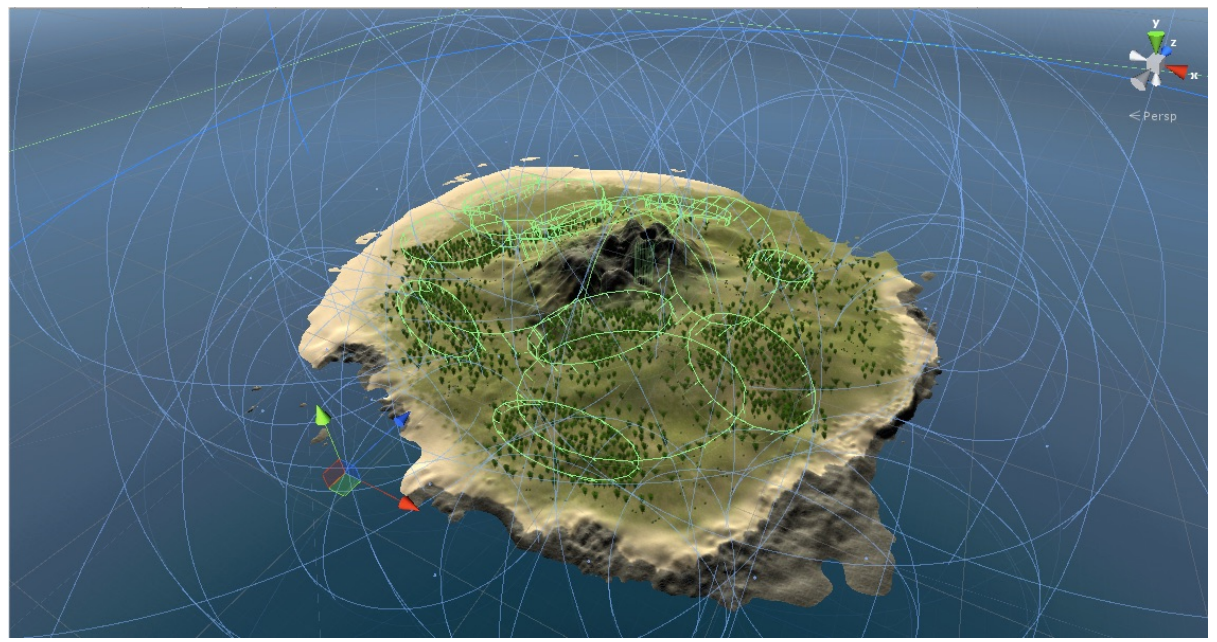

Fig 4. New Atlantis space "Ljubljana Island" showing reverb zones.

\section{First Reflections}

We have conducted some experiments concerning first reflections, that consisted of sending rays in all directions around the audio source, determining which rays hit a colliders (corresponding to an audio reflecting surface in our approximation), and then calculating the distance source-to-collider and collider-to-listener to define the 
audio delay for this path. The audio delay for each ray is then introduced in a multitap delay System (a FIR (Finite Impulse Response) filter) and applied to the audio source (using C\# OnAudioFilterRead() custom DSP code). Although still an approximation, this gave interesting results - a sense of scale, small spaces (with a short slap back echo) and big spaces (cliffs, canyons etc.). However at the time of writing we have not yet included this as a standard feature in NA.

Audio Sources Directivity and Roll-off

By default, the Unity audio engine does not provide management of audio source directivity. We have introduced the possibility to set a curve defining the source volume depending on the angle (similar to a polar diagram). Sound attenuation is implemented either by manual adjustment of roll off curves, where the builder defines how the sound attenuates over distance, or by using a physically accurate model for sound attenuation, (the well known inverse square distance law) where the builder simply defines the Sound Pressure Level at $1 \mathrm{~m}$ in dB (dB SPL).

\section{$5 \quad$ First Results - Student Workshops Public Presentations}

\subsection{Period September 2014 - January 2016 Description \& Method}

New Atlantis design and development took place between September 2014 and September 2015, during workshops in Chicago and Aix-En-Provence and through regular online (skype) meetings. Subsequently a workshop was organized in ENSCI les Ateliers in Paris that included a group of 15 students and the whole team of researchers, artists from Aix, Chicago, Troy and Paris. Within a week, students who had never worked with either sound or 3D environments were able to create a set of audiographic objects. At this point we programmed the 2016 performance (see below). From October 2015 to January 2016 the team worked separately but connectedly to prepare this performance: in Paris, another group of 10 students created their own objects, helped to design graphic interface elements such as the avatar and prepared for the upcoming performance. At the same time a Masters student from ESA-Aix (Alex Amiel) was in residence in SAIC Chicago, helping to build the Chicago space, a group of Masters students were working in ESA-Aix to create the Aix space and Ben Chang was working in RPI-Troy to create his own space. From January 10 to 15 another workshop with a fresh group of students from design art and gaming schools took place at Le Cube. This time, the framework was more advanced and the group was able to work and produce results much faster.

\subsection{Presentations January $16^{\text {th }} \mathbf{2 0 1 6}$}

For the performance on January 16th 2016 participants and audience were gathered in four different venues: Le Cube (Issy les Moulineaux, Paris France), the Vasarely Foundation (Aix en Provence), SAIC (Chicago) and Rensselaer Polytechnic Institute 
(Troy - New York state). The sound was played over 7.1 or 5.1 surround systems. As well as being connected via NewAtlantis the participants in different venues were connected via Skype during the performance and the audience was able listen to their commentaries and exchanges live. Five NA spaces were visited during the 80 -minute performance that started at $9 \mathrm{pm}$ in Paris and $2 \mathrm{pm}$ in Chicago. The audience reaction was enthusiastic and there was a positive reaction towards the prospect of continuing development.

\section{Conclusions and Further Research}

One of the aims of NA is to provide a platform for international academic, artistic, cultural and scientific exchange. In this sense the project has indeed succeeded in creating a robust bridge between the American and French research teams and students. The remaining challenge is for New Atlantis to become a real-time tool or platform for international artistic and musical collaboration. Hopes are high however since music and sound are after all internationally comprehensible. Possibly the most important step, that of making the app publicly available has yet to be made.

\subsection{Game architecture and Development}

With the ultimate goal of making all things audio in NA (i.e. that all visual elements have an audio counterpart) the concept of acoustic materials, could allow us to define the way a $3 \mathrm{D}$ surface interact with incoming sound, with parameters such as absorption at several audio frequencies, audio diffusion and reflection. We intend to implement audio path-finding using Unity's ray casting capabilities - precisely calculating the contribution of each source to each space and applying a chain of audio effects based on the traversed space's characteristics. This was implemented in the previous Panda3D/Pd version of NA it now has to be ported to Unity. Other advanced topics will be addressed in the future, such as accurate sound occlusion (possibly using ray casting between the source and the listener), and more advanced audio spatialization algorithms with the Unity Spatialization SDK. It is our intention to incorporate audio streaming capabilities into NA permitting the real time inclusion "real world" captured sounds. This would enable a voice object for example whereby a visitor could detach his or her voice (from navigation) and use it to make a distant space resound.

\subsection{Integration of and Intuitive synthesizer.}

Locus Sonus has recently joined with the audio group of the CNRS Laboratory LMA, now FRE PRISM with a program (accord cadre) funded by MCC (Ministry for Culture and Communication) and CNRS (Centre Nationale pour la Recherche Scientifique). In this context it is planned to integrate the LMA's research into intuitive synthesizer interaction. In effect this synthesizer that is controlled by 
"semantic descriptions of sound events"[34][35], including non-existent ones, would appear to be an ideal solution for audio graphic experimentation in NA.

\subsection{Interface and Graphic Design}

Before releasing a public version of the New Atlantis app in is necessary to improve the ergonomics of the user interface. The design process is programmed and we hope to achieve this goal within the coming months.

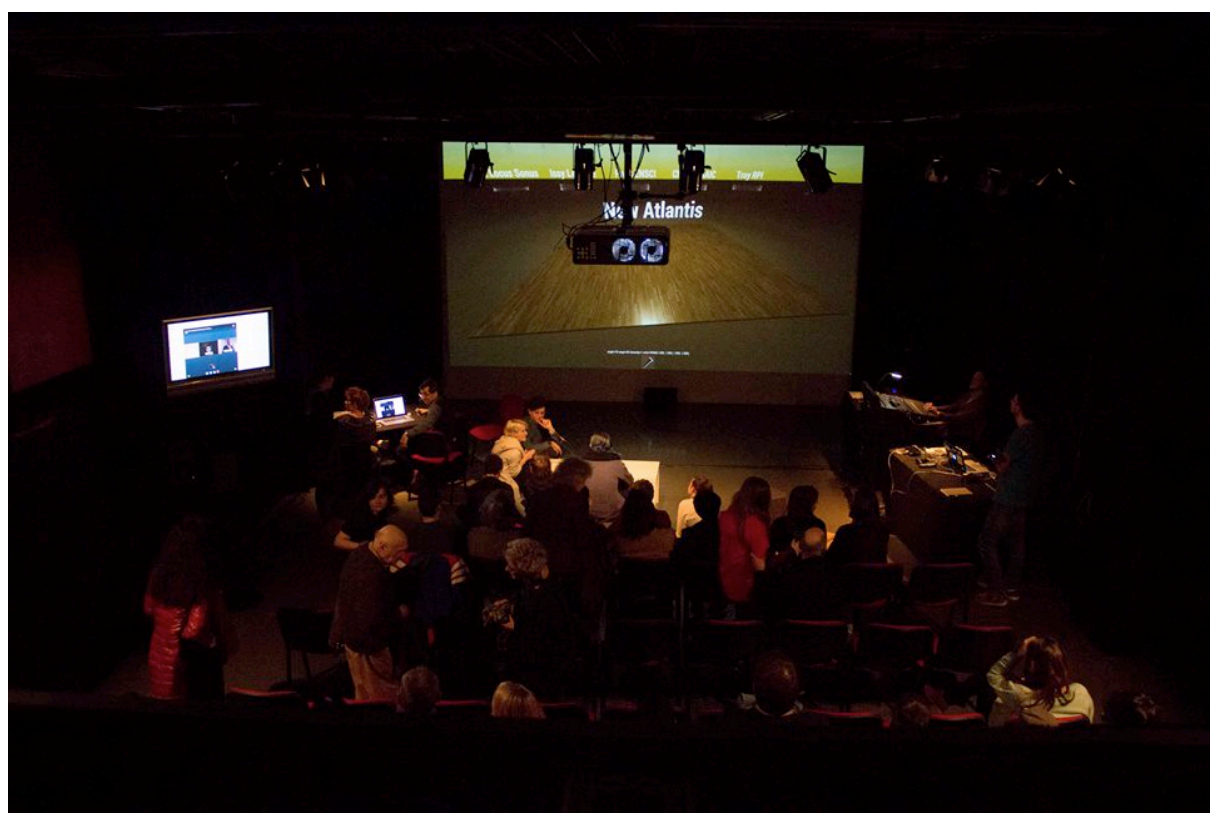

Fig 5. New Atlantis performance synchronized between: Le Cube, Vasarely Foundation, SAIC and RPI. January 2015. Photo: Le Cube. 


\section{$7 \quad$ New Atlantis Team 2016}

\section{Coordination:}

Peter Sinclair (Locus Sonus - ESAAix), Peter Gena (SAIC), Roland Cahen (ENSCI)

Development

Jonathan Tanant (JonLab) : lead developer and software architect, Components developement :

Alexandre Amiel (ESAAix)

Faculty/Research:

Mark Anderson (3d Graphics SAIC), Robb Drinkwater (Audio programming SAIC), Michael

Fox (3d GraphicsSAIC), Jerome Joy (Audio Locus Sonus)

Ben Chang (Programming, 3d Graphics)

\section{Students:}

Daan De Lange, Théo Paolo, Alexandre Amiel, Antoine Langlois (ESAAix) Adrien Giordana, Luca Notafrancesco, Marion Talou, Dorian Roussel, Valentin Moebs, Anaïs Maurette de Castro, Thomas Signollet, Oscar Gillet, Juliette Gueganton, Mathilde Miossec, Gamzar Lee, David Guinot, Paul Barret, Gaëtan Marchand, Aristide Hersant, Louis Fabre, Blanche Garnier, Lucas Dubosque, Alexandra Radulescu.

Organization:

Anne Roquigny (Locus Sonus), Julie Karsenty (ESAAix)

Partners: Locus Sonus ESAAix Ecole supérieure d'art d'Aix-en-Provence

ENSCI Les Ateliers, Rensaler Polytechnic Institute, Troy, School of the art Institute of Chicago, Chicago USA

\section{Previous Participants:}

Ricardo Garcia, Gonzague Defos de Rau, Margarita Benitez, Anne Laforet, Jerome Abel, Eddie Breitweiser, Sébastien Vacherand.

\section{References}

1. Bacon, F.: The New Atlantis. no publisher given, United Kingdom (1628)

2. French-Americain Cultural Exchange, http://face-foundation.org/index.html

3. Second Life official site, http://secondlife.com/

4. Pure Data - Pd community site, https://puredata.info/

5. LS-SL Seconde Nature, http://www.secondenature.org/LS-SL-LOCUS-SONUS-INSECOND-LIFE.html

6. Panda3D - Free 3D game Engine, https://www.panda3d.org/

7. NA version1, http://locusonus.org/w/?page=New+Atlantis

8. LaForet, A.: New Atlantis, un monde virtuel sonore. In Locus Sonus 10 ans d'expérimentation en art sonore. pp. - Le Mot et Le Reste, Marseille (2015)

9. Unity Game Engine, https://unity3d.com/

10. AudioGaming, http://www.audiogaming.net/game-sound-design

11. UPIC https://en.wikipedia.org/wiki/UPICÒ

12. Cage, J. : Fontana Mix. Peters, New York, $n^{\circ}$ EP 6712, (1960) 
13. Sinclair, P., Joy, J.: Locus Sonus 10 ans d'expérimentation en art sonore. pp. - Le Mot et Le Reste, Marseille (2015)

14. Polymeneas-Liontiris. T, Loveday-Edwards A. (2013) The Disklavier in Networked Music Performances In: ATINER, $4^{\text {th }}$ Annual International Conference on Visual and Performing Arts 3-6 June 2013, Athens: Greece.

15. Sinclair, P.: ed. Sonification - What Where How Why. AI \& Society (Springer) 27, no. 2 (05 2012).

16. Installation Icare de Ivan Chabanaud, http://www.musicvideoart.heureexquise.org/video.php?id=2151

17. BANDONEON, http://roland.cahen.pagesperso-orange.fr/bandoneon/Bandoneon.htm http://www.edit-revue.com/?Article=200

18. Cahen, R.: Sound Navigation in the PHASE installation: producing music as performing a game using haptic feedback. Subsol, Gérard, ed.

19. ENIGMES, http://projetenigmes.free.fr/wiki/index.php?title=Accueil

20. TOPOPHONIE, http://www.topophonie.com

21. UDKOSC, https://ccrma.stanford.edu/wiki/UDKOSC

22. Avatar Orchestra Metaverse, http://avatarorchestra.blogspot.fr/

23. (btween 22 and 23) Key Ed., Kanaga D.: Proteus - A game by Twisted Trees, http://twistedtreegames.com/proteus/

24. Jacques Tati: Composing in Sound and Image, https://www.criterion.com/current/posts/3337-jacques-tati-composing-in-sound-and-image

25. Cahen, R. Rodet, X. Lambert, JP.:Virtual Storytelling: Using Virtual Reality Technologies for Storytelling: Third International Conference, ICVS 2005, Strasbourg, France, November 30-December 2, 2005: Proceedings.

26. Sinclair, P.: Inside Zeno's Arrow: Mobile Captation and Sonification Audio Mobility Vol. 9 No. 2. 2015, http://wi.mobilities.ca/

27. Thibaud, JP.: Towards a praxiology of sound environment. Sensory Studies Sensorial Investigations, 2010, pp.1-7.

28. Schafer, RM.: The Tuning Of The World. Alfred Knopf, New York, (1977)

29. Key Ed., Kanaga D.: Proteus - A game by Twisted Trees,

http://twistedtreegames.com/proteus/

30. Locus Sonus Sound Map, http://locusonus.org/soundmap/051/

31. Locus Sonus, http://locusonus.org

32. Cahen, R.: Teaching Sound--Design @ENSCI les Ateliers, Sound-Design and innovation: a virtuous circle. Cumulus 2015 Proceedings

33. SAIC, http://www.saic.edu/academics/departments/ats/

34. Conan S., Thoret E., Aramaki M., Derrien O., Gondre C., Kronland-Martinet R., Ystad S. (2014). An intuitive synthesizer of continuous interaction sounds: Rubbing, Scratching and Rolling. Computer Music Journal, 38(4) 24-37, doi:10.1162/COMJ_a_00266

35. Pruvost L., Scherrer B., Aramaki M., Ystad S., Kronland-Martinet R. Perception-Based Interactive Sound Synthesis of Morphing Solids' Interactions. Proceedings of the Siggraph Asia 2015, Kobe, Japon, 2-5 Novembre 2015. 